\title{
Local Minimization Paradigm in Numerical Modelling of Foraminiferal Shells
}

\author{
Paweł Topa ${ }^{1}$, Jarosław Tyszka ${ }^{2}$ \\ 1 Institute of Computer Sciences, University of Mining and Metallurgy, al. \\ Mickiewicza 30, 30-059 Kraków, Poland \\ email: topa@uci.agh.edu.pl \\ 2 Institute of Geological Sciences, Polish Academy of Sciences, Cracow Research \\ Centre, ul. Senacka 1, 31-002 Kraków, Poland \\ email: ndtyszka@cyf-kr. edu.pl
}

\begin{abstract}
We present a new approach to modelling of foraminiferal shells. Previous models referred to fixed reference axes and neglected apertures, which play a crucial role in morphogenesis of shells. Our 2D preliminary model applies the moving reference system based on introducing of apertures and minimization of the local communication path (LCP). LCP defines the position of every final aperture. A formal description of simple analytical methods with some elements of randomness is given in this paper. Selected examples of simulated shells are figured.
\end{abstract}

\section{Introduction}

The emergence of forms in the growth process of foraminiferal shells is the essential problem in ontogenesis of these creatures. Foraminifera are singlecelled organisms (protozoans) that construct shells. They inhabit all marine and marginal marine environments from very shallow to the deep ocean floor. Depending on the group, the shell may be made of organic compounds, sand grains and other particles cemented (agglutinated) together, or secreted from crystalline calcium carbonates. Foraminifera are a unique group of autonomous unicellulars reaching a size class (typically $0.1 \mathrm{~mm}$ to $1 \mathrm{~mm}$ in size, up to $20 \mathrm{~cm}$ ) which is characteristic of small metacellular organisms [1]. Foraminiferal shells occur in an enormous variety of shapes (Fig.1). The majority of foraminifera are built of chambers, which are cavities containing the protoplasm surrounded (enveloped) by a firm wall [2]. The shape of a shell results from growth processes and depends on a chamber form, location and the type of aperture as well as the final chamber arrangement (Fig.1).

Modelling of foraminifera started very early with the classical work of Berger [3]. Nevertheless, so far simple regular morphologies (e.g. planispiral, helicoidal, uncoiled) that do not express complexity of foraminiferal shell patterns have only been simulated. It is therefore necessary to find a new alternative approach for further progress in theoretical morphospace of these organisms. The general aim is to gain a better understanding of foraminiferal shell morphology and its 
incredible variability, and thus, to find essential geometric growth rules. Systematics of foraminifera is based on shell morphology (shell composition and microstructure, chamber form and arrangement, aperture type etc.). Theoretical shell morphology can help to verify some taxonomic rules. This study briefly summarizes previous foraminiferal models and presents a new approach to model basic morphologies.

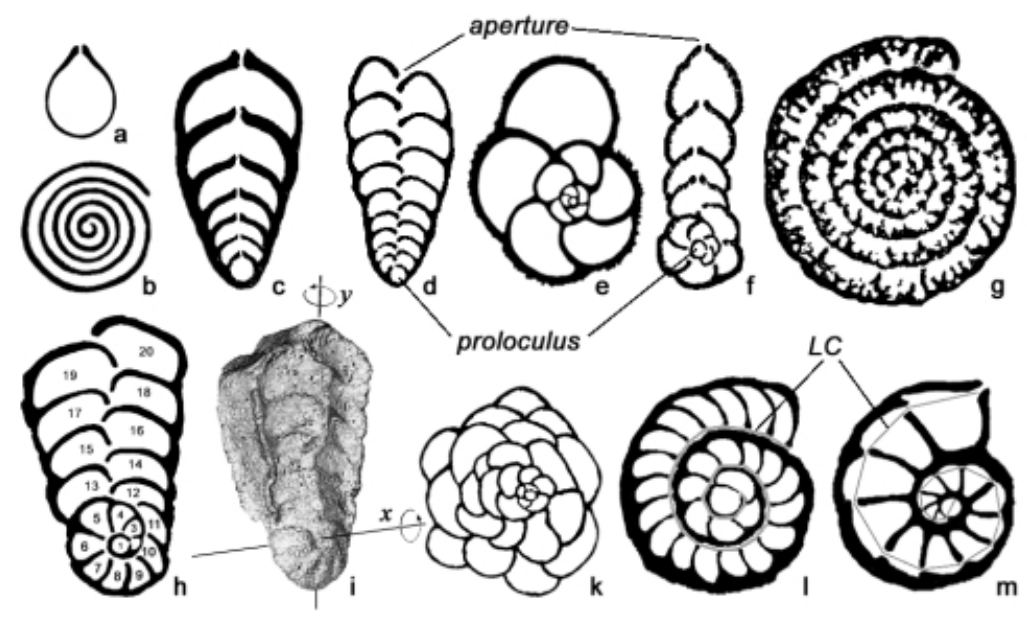

Fig. 1. Cross sections of foraminiferal shells; (a) Fissurina, unilocular shell; (b) Spirillina, non-septate bi-chambered; (c) Pseudonodosaria, multilocular uniserial shell; (d) Bolivina, biserial; (e) Globorotalia low helicoidal; (f) Ammobaculites, planispiral becoming uniserial; (g) Reticulinella, complex foraminifer with multiple apertures (not expressed here); (h, i) Heterantyx, planispiral switching to biserial; first 12 chambers rotate along the ' $x$ ' axis, then (chambers 13-20) along the ' $y$ ' axis, SEM view; (k) Planorbulina low helicoidal to random coiling; (l) Miscellanea, planispiral with the shortest global communication path (LC) via foramina (m) Lenticulina, planispiral shell with the longest global communication path (LC). Not to scale, actual size range from 0.1 to $1.3 \mathrm{~mm}$.

\section{Overview of foraminiferal modelling}

Foraminiferal shells can be divided into 3 informal groups corresponding to the trend of increasing complexity (Fig. 1): (i) unilocular shells (Fig. 1a); (ii) multilocular shells (multichambered forms) (Fig. 1b-m); (iii) complex shells (multilocular shells with chambers divided into smaller chamberlets and/or having complex wall structure) (Fig. 1g).

Multilocular foraminifera enlarge in discrete growth processes of serial chamber additions successively united into the shell during ontogeny (Fig. 1c-m). The first usually globular chamber is called proloculus. The opening on every 
last chamber, through which pseudopodia (rhizopodia) extrude, is termed the aperture. Adjacent chambers are separated by septa but connected by foramina (Fig. 1c-m). Another type of foraminiferal morphology is produced by tubular chambers (Fig. 1b), which show a different growth pattern closely related to accretive growth of mollusks and/or branching system of plants (see [4], [5] for overview).

Over three decades ago, Berger [3] in his pioneering work already created the first theoretical morphospace of foraminifera. The theoretical morphospace has three parameters which define simple step-by-step rotation of a circle with a certain overlap and expansion of circle radius. This model simulates isometric growth (all three parameters are held constant through ontogeny) and is confined to planispiral and trochospiral shells composed of spherical chambers (Fig. $1 \mathrm{e}, 1)$.

Signes et al. [6] designed a similar three dimensional theoretical morphogenetic model with two basic assumptions concerning foraminiferal growth: the shape of the chambers in the shell remains constant with growth, and the volume of each new chamber increases in a constant proportion to the pre-existing volume of the shell. This model produces isometric growth with coiling in a fixedreference frame, which is similar to Raup's [7] and Berger's [3] models. Webb and Swan [8] partly extended this classical theoretical morphospace to uncoiled morphologies based on the different definition of the angle between successive chambers, which was not referred to the center of the shell.

Some other authors also simulated allometry of foraminiferal shells. Brasier [9] who produced a working morphospace model of foraminiferal form using four parameters, which actually correspond to Berger's parameters expanded by the degree of extension of growth along coiling axis and the degree of chamber compression and overlap. This last parameter includes allometry into the system. Another important difference is that chambers are not directly rotated but translated. An interesting model was presented by De Renzi ([10], [11]) who simulated allometric growth of some larger foraminifera based on the logistic model applied in polar coordinates.

\section{New approach}

All the mentioned simulations use theoretical axes, which have no morphogenetic or physiological meaning. Chambers (circles or spheres) are only rotated and translated along these artificial axes, which are fixed and serve as a reference line for growth process. Therefore, these models can simulate plane planispiral, trochospiral (helicospiral) or uniserial chamber arrangement, but cannot simulate more complex patterns. For instance, they cannot model gradual or abrupt changes of coiling axis that cause different chamber arrangements (Fig. 1f, h, i, k).

It seems to be clear that it is necessary to give up the fixed-reference frame based on theoretical coiling axes in favour of moving-reference system. In general, 
the moving reference model is based on simple principles of motion and stepwise growth [12]. At each growth step, the aperture migrates from its present position to a new position, according to locally defined rules [12]. Such models have been developed for simulation of ammonites. Okamoto [13] proposed a tube model for all types of shell coiling, including heteromorph forms with abrupt changes of coiling pattern. His approach integrates accretional growth of the aperture (opening of the shell) without defining any fixed coordinate system. Similar moving-reference frame is used in simulating radiate accretive growth of marine sessile organisms, such as corals and sponges, where growth axis is associated with the local maximum of growth (e.g. [14]). A comparable approach is also used in simulation of plant growth [15].

In order to define a moving-reference system for modelling of foraminifers, it is also reasonable to use apertures, which seem to be essential for location of a new growing chamber. Analyses of different modes of chamber growth in foraminifera suggest that the position of the aperture controls local chamber arrangement (see Fig. 1c-m). The problem is that although the processes associated with chamber formation are relatively well understood, we still know very little of how foraminiferal apertures are formed in nature.

Hottinger [16] discerned, the foraminifer must devise methods to shorten distances between the first and last compartments of its shell. Brasier [17] also analysed the energetics of protoplasmic pathways through the organism and concluded that foraminifers show the trend towards minimizing distance from the back of the first chamber (proloculus) to the most proximal aperture in the final chamber (Fig. 11). He standardized this cumulative distance and named it MinLOC (minimum line of communication).

This 'rule' seems to be valid for many foraminiferal architectures, but not for all of them. Lenticulina and other coiled lagenids are curious exceptions to this rule because the foramina are located at the outer margin of the chamber [16] that creates the longest possible global line of communication (Fig. 1m). Nevertheless, local distances between adjacent foramina are in fact minimal. Thus, it is a paradox that the shortest distance between adjacent chambers creates the longest global line of communication between the first and last chambers. The conclusion is that the "the growth programme" of a foraminifer does not directly control cumulative arrangement of chambers. It does control formation of every chamber itself and optimisation (minimization) of the local distance between the last and the new-formed aperture of a new chamber. This minimization of the local communication path (LCP) seems to be more general (even if it is not the only rule) and should help to define simulated apertures.

\section{Model}

The model, we present in this paper, is significantly simplified. We have limited our study to a 2D case only. The modelling in 3D is more complicated and not necessary at this stage of work. The chambers are represented by regular circles. Although, real chambers can have very diverse shapes, the shells with 
spheroidal chambers are relatively frequently occurring. Additionally, we can apply simple analytical methods to compute the aperture location.

The modelling of development of foraminifera consists of discrete steps in which successive chambers are added to the forming shell. The position of the newly created chamber depends on the aperture location of the previous chamber and so called "vector of growth" (see Fig. 2). "Vector of growth" is attached at the aperture of the chamber $c_{i}$ and pointed at the center of chamber $c_{i+1}$. Thus, in fact the aperture represents the moving reference point and the vector indicates the current direction of growth. The aperture of the new chamber is calculated according to the minimization principle. The distance between the apertures of the two successive chambers must be as short as possible. The apertures cannot be enclosed within any other already existing chamber. The most exterior aperture must be connected with the first chamber (proloculus) by a line of communication running through all the previous apertures (foramina). Fig. 3 presents a few hypothetical successive steps of simulation of ontogenetic shell development.

For convenience, calculations are made in circular co-ordinates. Instead of

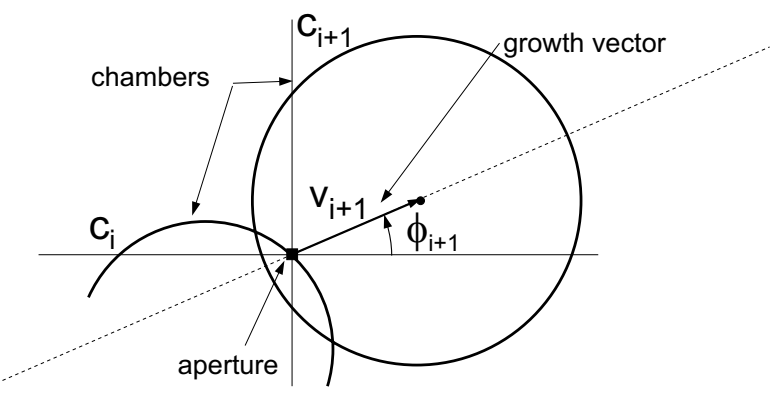

Fig. 2. Vector of growth

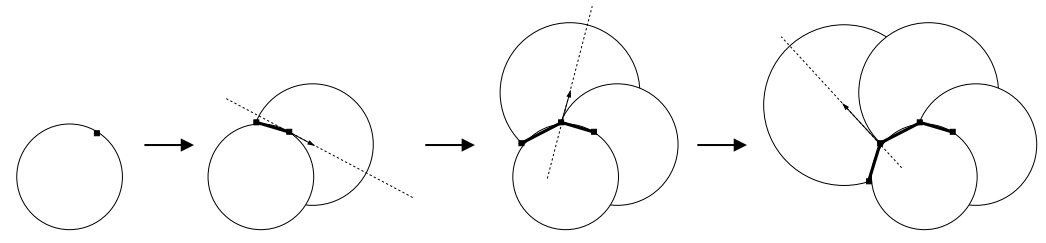

Fig. 3. Modelling the development of foraminferal shell

euclidian $x, y$ coordinators we use the length of vector $\left|\boldsymbol{v}_{i+1}\right|$ and angle $\phi_{i+1}$ (see Fig. 2).

We define the two parameters, which control the development of shell: 
1. GF - chamber growth ratio: the ratio between the sizes (radius) of two successive chambers,

2. $V\left(\Phi, R_{v}\right)$ - vector of growth ( $\Phi$ and $R_{v}$ stand for it polar coordinates).

All the previous models assume invariance of their parameters during the modelling of the shell development. In our model, the foregoing parameters do not have to be constant and they can fluctuate within the ranges, we specify. The chamber growth ratio and growth vector are chosen at random within the given range. In such a way we can model the natural susceptibility of some species to forming a specific shell pattern. Specifying the exact values instead of the ranges of fluctuations, let us partly track models delivered from the fixed reference system.

Our algorithm works as follows:

Size and position of the aperture of the first chamber are set arbitrarily as the starting parameters of the simulation. In loop, the following operations are performed:

1. the position of the new chamber is calculated:

- the radius of the new chamber $\left(r_{i+1}\right)$ fluctuate within the given range adjacent to the mean value estimated on real foraminifera.

- the growth vector $\boldsymbol{v}_{i+1}$ is calculated at random.

2. the position of the new aperture is calculated (see Fig 4):

- we minimize the distance between the apertures of the two successive chambers - $\min \left(\left|\boldsymbol{u}_{i+1}\right|\right)$ (see appendix A),

- the new aperture cannot be placed within any previously created chamber.

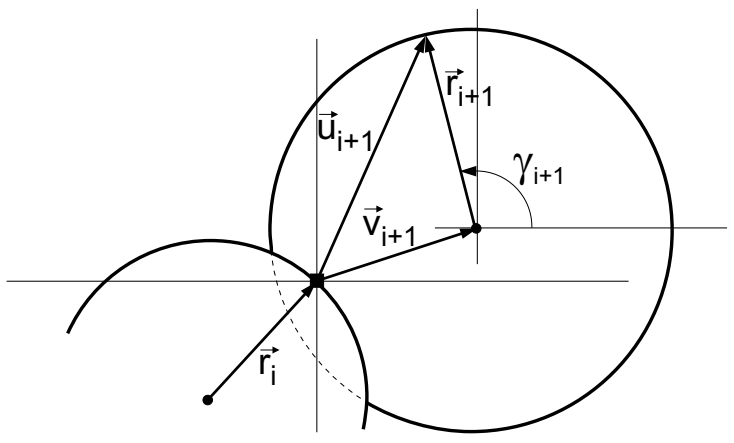

Fig. 4. Calculating the aperture location

The area, in which the aperture cannot be located, is determined by calculating the points, in which the circle of the new chamber is crossing over all the previous chambers. 
The simplifications we have made, let us solve the minimization problem in an analytical way. Detailed description of the used mathematical methods can be found in Appendix A.

\section{$5 \quad$ Results}

Fig 5 presents the forms generated by using our models. The three first pictures have been supplemented by the numbering of successive chambers and the communication line connecting its apertures. This line usually crosses every previous chamber, which is another simplification, not affecting our general model.

Simulated shells show either relatively stable growth pattern (Fig. 5a, b, d),
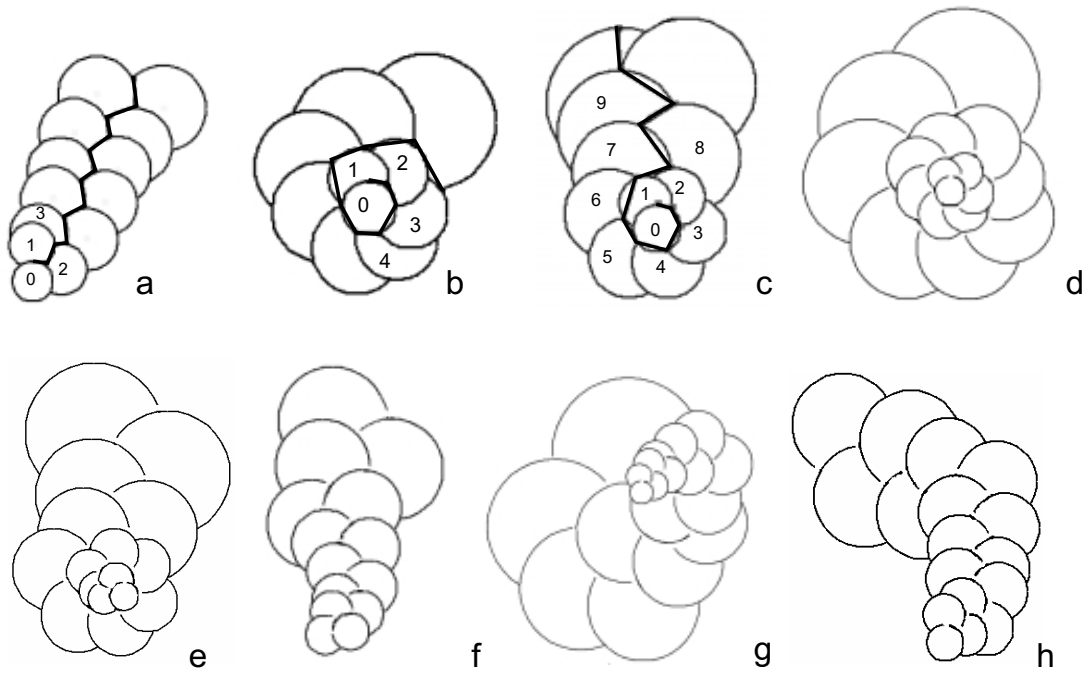

Fig. 5. Shells generated by computer; (a,f) biserial form; (b,d) spiral form; (c, e) initially spiral, after 7 chambers changes to biserial; $(g, h)$ alternately biserial and spiral.

or abrubtly change the mode of coiling (Fig. $5 \mathrm{c}, \mathrm{e}, \mathrm{g}, \mathrm{h}$ ). Most of the simulated shell forms do have real counterparts (compare Fig 1). Some forms mimic abnormal shells usually related to environmental stress (Fig. 5f, h). Another set of generated shells is very chaotic and flexible to growth switch-overs (Fig. 5g). These shells may resemble some real attached (adherent) foraminifera, as well as some irregular (in 3D) agglutinated foraminifera.

We observe that ranges of fluctuations of the growth vector $V\left(\Phi, R_{v}\right)$ are crucial for generating forms. The narrow range of $\Phi$ and longer $R_{v}$ results in elongated, biserial forms (Fig. 5a,f), while wider range of $\Phi$ and short $R_{v}$ lead to formulation of spiral shells (Fig. $5 \mathrm{~b}, \mathrm{~d}$ ). Other combinations of these parameters usually follows to mixed forms (Fig. 5c,e,g,h). 


\section{Conclusions}

A new approach to modeling of foraminiferal shells has been presented. Previous models referred to fixed axes and neglected apertures [3], [6]. Our 2D simple model applies the moving reference system which has already been described to simulate heteromorphic shells of ammonites [13], [12]. This system is based on introducing of apertures as reference points, which in reality and in our model are responsible for emplacement of every new chamber. Minimization paradigm of the local communication path (LCP) helps to define an aperture in every newly added chamber. LCP rule derives from previous studies [16], [17] suggesting global shortening of the distance between the first and the last chamber via internal foramina and an external aperture. This rule seems to be based on local optimization during formation of a new chamber. Even if it does not explain all the cases, it is a closer approximation of actual morphogenetic constraints during the step-by-step growth of foraminiferal shells.

In order to imitate reality, some elements of randomness, as another novelty in foraminiferal modeling, are applied. This approach seems to be very promising for further studies. It may mimic random genetic variability (mutations) and influence of external (environmental) factors.

We are aware of numerous oversimplifications introduced into the model. Some of them can be overcome based on the same analytical approach. Twodimensional simulations have only limited value, because foraminifera grow in $3 \mathrm{D}$ and most of the growth patterns cannot be reduced to two dimensions. Nevertheless, the moving reference system and the minimization paradigm applied here can be simply transferred to three-dimensional space.

Spherical shape of chambers also tremendously reduces variability of chamber shapes in different taxa. Furthermore, many foraminifers change shape of chambers during ontogenesis, their growth is often strongly allometric. It is clear that we have to test other methods (under study) to incorporate chamber shape into the model. A first approximation could be an introduction the implicit surfaces (blobs, metaballs)[18] to model chambers with irregular shapes. An overall form of the whole specimen directly depends on cumulative succession of chambers. Single apertures per chamber represent another simplification of real shell variability. Multiple apertures will be essential for future attempts to simulate complex foraminiferal shells.

Our model does not require high performance computing. An analytical method of optimization makes that the most difficult calculations are made on paper (see Appendix A). Moving our method to 3D with simple spherical chambers would complicate the paper calculations, but the computational cost will be kept on the same level. Thanks to this, we can implement presented model in Java language as an applet (with Java 3D as a graphic library) and published it on WWW page for broader audience of researchers interested in morphogenetic studies. Models with chambers represented by implicit surfaces, will require advanced numerical optimization methods and they will be more demanding for computational power. 


\section{Acknowledgments}

We are grateful to Drs Sheila J Stubbles, Witold Alda, Mariusz Paszkowski and two anonymous reviewers for their contribution to this paper.

\section{A Distance minimization}

We minimize the length of the vector $\boldsymbol{u}_{i+1}=\boldsymbol{v}_{i+1}+\boldsymbol{r}_{i+1}$ (see Fig. 4).

$$
\begin{gathered}
\left|\boldsymbol{u}_{i+1}\right|=\sqrt{\left(x v_{i+1}+x r_{i+1}\right)^{2}+\left(y v_{i+1}+y r_{i+1}\right)^{2}}= \\
\sqrt{x v_{i+1}^{2}+2 x v_{i+1} x r_{i+1}+x r_{i+1}^{2}+y v_{i+1}^{2}+2 y v_{i+1} y r_{i+1}+y r_{i+1}^{2}}
\end{gathered}
$$

Let's make the following assumption:

The coordinators of vector $\boldsymbol{v}_{i+1}$ are known therefore we can simplify our formula by introducing variables $a=x v_{i+1}$ and $b=y v_{i+1}$ :

Lets express vector $\boldsymbol{r}_{i+1}$ in polar coordinators:

$$
x r_{i+1}=r_{i+1} \cos \gamma_{i+1}, \quad y r_{i+1}=r_{i+1} \sin \gamma_{i+1}
$$

The radius $r_{i+1}$ is also known, so we can introduce variable $r: r_{i+1}=r$. The angle $\gamma_{i+1}$ must be calculated.

Now, the formula 1 has form:

$$
\sqrt{a^{2}+2 a r \cos \gamma_{i+1}+r^{2} \cos \gamma_{i+1}^{2}+b^{2}+2 b r \sin \gamma_{i+1}+r^{2} \sin \gamma_{i+1}}
$$

We reduce this formula to the form:

$$
\sqrt{c+2 r\left(a \cos \gamma_{i+1}+a \sin \gamma_{i+1}\right)}, \text { where } c=a^{2}+b^{2}+r^{2}
$$

We can treat this formula as a function $f\left(\gamma_{i+1}\right)$. The first derivative of the function is:

$$
f^{\prime}\left(\gamma_{i+1}\right)=\frac{2 r\left(b \cos \gamma_{i+1}-a \sin \gamma_{i+1}\right)}{2 \sqrt{c+2 r\left(a \cos \gamma_{i+1}+b \sin \gamma_{i+1}\right)}}
$$

The left side of the equation must be equated to zero.

$$
\frac{2 r\left(b \cos \gamma_{i+1}-a \sin \gamma_{i+1}\right)}{2 \sqrt{c+2 r\left(a \cos \gamma_{i+1}+b \sin \gamma_{i+1}\right)}}=0
$$

The $\gamma_{i+1}$ value must preserve the following conditions:

$$
\begin{gathered}
2 r\left(b \cos \gamma_{i+1}-a \sin \gamma_{i+1}\right)=0 \\
2 \sqrt{c+2 r\left(a \cos \gamma_{i+1}+b \sin \gamma_{i+1}\right)} \neq 0
\end{gathered}
$$

Solving the equation 5 we obtain the points in which function $f\left(\gamma_{i+1}\right)$ achieve extremal values:

$$
\gamma_{i+1}=\arctan \frac{b}{a}+k \pi, \quad \text { where } \quad k=0,1,2 \ldots
$$



lated:

Returning to the original nomenclature of variables, the $\gamma_{i+1}$ can be calcu-

$$
\gamma_{i+1}=\arctan \frac{y v_{i+1}}{x v_{i+1}}+k \pi, \quad \text { where } k=0,1,2 \ldots
$$

$\gamma_{i+1} \in\langle 0,2 \pi)$. To calculate the angle in which the function achieve minima, we examine the points: $\arctan \frac{y v_{i+1}}{x v_{i+1}}$ and $\arctan \frac{y v_{i+1}}{x v_{i+1}}+\pi$.

\section{References}

[1] Hottinger L., Functional morphology of benthic foraminiferal shells, envelopes of cells beyond measure, Micropaleontology, 46:supplement no. 1, pp. 57-86, 2000.

[2] Lipps J.H., Fossil Prokaryotes and Protists, Blackwell, Boston, 1993.

[3] Berger W.H., Planktonic foraminifera: basic morphology and ecologic implications., Journal of Paleont., 6(43):1369-1383, 1969.

[4] Fowler D.R., Meinhardt H., Prusinkiewicz P., Modeling seashells, Proceedings of SIGGRAPH'92. Computer Graphics, 26(2):379-387, 1992.

[5] McGhee Jr., G.R., Theoretical Morphology. The Concept and its Application. Perspectives in Paleobiology and Earth History, Columbia University Press, New York, 1999.

[6] Signes M., Bijma J., Hemleben C., Ott R., A model for planktic foraminiferal shell growth, Paleobiology, 19:71-91, 1993.

[7] Raup D.M., Geometric analysis of shell coiling: General problems, Journal of Paleontology, 40:1178-1190, 1966.

[8] Webb L.P., Swan A.R.C, Estimation of parameters of foraminiferal test geometry by image analysis, Paleontology, 39:471-475, 1996.

[9] Brasier, Microfossils, Allen \& Unwin, London 1980.

[10] De Renzi M., Shell coiling in some larger foraminifera: general comments and problems, Paleobiology, 14(4):387-400, 1988.

[11] De Renzi M., Theoretical morphology of logistic coiling exemplified by tests of genus Alveolina (larger foraminifera), Neues Jahrbuch für Geologie und Paläontologie. Abhandlungen, 195:241-251, 1995.

[12] Ackerly S.C, Kinematics of accretionary shell growth, with examples from brachiopods and molluscus Paleobiology, 15:147-164, 1989.

[13] Okamoto T., Analysis of heteromorphy ammonoids by differential geometry, Palaeontology,31:35-52, 1988.

[14] Kaandorp J.A., A formal description of radiate accretive growth J. theor. Biol., 166:149-161, 1994.

[15] Prusinkiewicz P., Lindenmayer A., The Algorithmic Beauty of Plants, SpringerVerlag, New York, 1990.

[16] Hottinger L., Comparative Anatomy of Elementary Shell Structures in Selected Larger Foraminifera, In: Hedley, R.H. and Adams, C.G. (eds.) Foraminifera, 3:203-266, 1978.

[17] Brasier, Foraminiferid architectural history; a review using the MinLOC and PI methods, Journal of Micropalaeontology, 1:95-105, 1982.

[18] Opalach A., Maddock S., Introduction to Modelling and Animation using Implicit Surfaces, Overview of Implicit Surfaces. Course Notes No 3, pp 1.1-1.13, 1995. 